\title{
Artificial Intelligence to Combat the Sting of the Pandemic on the Psychological Realms of Human Brain
}

\author{
Mary Fabiola Sagayaraj ${ }^{1}$ (]) $\cdot$ Ignisha Rajathi George ${ }^{2} \cdot$ R. Vedhapriyavadhana ${ }^{3} \cdot$ L. R. Priya ${ }^{4}$
}

Received: 24 September 2021 / Accepted: 6 January 2022 / Published online: 7 March 2022

(c) The Author(s), under exclusive licence to Springer Nature Singapore Pte Ltd 2022

\begin{abstract}
We are inhabitants of the universe in this anomalous time due to the novel corona virus. COVID-19, which WHO has annotated as a pandemic, is an infectious and contagious disease hastened by the most freshly perceived coronavirus. COVID-19 has gravely hit different people in different ways, some with physical symptoms and some patients will likely be more susceptible to insignificant and extreme symptoms of mental illness. Such patients often with pre-existing mobility limitations get imprisoned in their own homes. The current pandemic beseeches for intense contemplation of the mental health of patients to reduce their worry and heal their fear, depression and anxiety due to quarantine. Artificial intelligence (AI) is more and more remarkable in public, academic, and clinical provinces. This paper aims in addressing the mental health problems faced by patients affected by COVID-19 and recommends artificial intelligence integrated virtual counsellor who can provide advice to their problems. The strategy of AI is being developed and enhanced which will possibly help in addressing the problems in this pandemic time.
\end{abstract}

Keywords Coronavirus · Symptoms · Stress · Anxiety · Depression · Isolation · Mental health $\cdot$ Lockdown · Fear . Quarantine $\cdot$ Pandemic $\cdot$ COVID-19 $\cdot$ Artificial intelligence

This article is part of the topical collection "Intelligent Systems" guest edited by Geetha Ganesan, Lalit Garg, Renu Dhir, Vijay Kumar and Manik Sharma.

Mary Fabiola Sagayaraj

fabiola.mary@gmail.com

Ignisha Rajathi George

docignisharajathi@gmail.com

R. Vedhapriyavadhana

vedhapriya.vadhana@gmail.com

L. R. Priya

lrpriya11@gmail.com

1 Department of Science and Humanities, Sri Krishna College of Engineering and Technology, Coimbatore 641008, India

2 Department of Computer Science and Engineering, Sri Krishna College of Engineering and Technology, Coimbatore 641008, India

3 School of Computer Science Engineering, Vellore Institute of Technology, Chennai, India

4 Department of Electronics and Communication Engineering, Francis Xavier Engineering College, Tirunelveli, India

\section{Introduction}

To reduce or break the escalation of infection, quarantine or physical isolation was practiced for centuries. The novel coronavirus COVID-19 was initially reported in Wuhan, China, in late 2019 and has expeditiously spread across the globe becoming a pandemic and has hit India off late [1]. Current quarantine policies have been thrusted world-wide aiming to lessen the spread of the COVID-19 infection including lockdowns, voluntary home quarantine, and restriction on the assemblies, social and public events. These restrictions imposed due to COVID-19 outbreak, have caused significant chaos to individuals, families, communities and the whole world. Much of world's population have been affected extremely altering and complicating features of daily life that were easy and direct [2].

The precariousness is more and more testing psychological resilience of the people. When the universal cynosure has mostly been on testing, discovering a cure and avert communication of the disease; in the midst of all these issues people are also experiencing a multitudinous psychological problem in regulating to the current way of life and panic caused by the pestilence. 
A shift in the usual life makes people feel fearful and unsafe which can be linked with the disease-fear contagion chain because of the unawareness of the cause or progression of the disease and outcomes, false news and trumpedup story which can lead to prejudice. Social support is the need of the hour in times of dreadful situations like this. Hence, imposed quarantine or isolation strategy can pressurize an individual's sense of association and can lead to a profuse effect on mental health [3].

Social isolation interconnected with quarantine can be the major impulse for many mental health effects [4]; even in people with proper mental health. These can involve desperate stress disorders, sleeplessness, emotional pain and mind disorders, agitation and trauma because of financial perturbation, disappointments, weariness, rejection and poor communication [5]. All these mental health troubles can be solved by prognosticating it at the earliest and provide them virtual therapists or counsellors to whom they can open out easily. Artificial intelligence can recognize and identify such persons and avert the growth of severe mental illness by providing proper counselling or therapy [6].

The novel coronavirus has risen as a major health disaster [7]. This began as an outbreak in India from January 29, and has rapidly increased with 40,00,000 confirmed cases, $30,37,106$ recovered number and death rate of 68,598 till the month of September as per the statistics provided by Statista, Health and pharmaceuticals, State of Health on the topic 'Cumulative of the coronavirus (COVID-19) confirmed, recovered and deceased numbers across India from January to September 2020' WHO and the other global health establishments have constantly emphasized the significance of "reducing the curve" to manage the pandemic as shown in Fig. 1.

\section{Threat of COVID-19 and the Fluctuating Psychology}

The fear of coronavirus can crumple our psychological reactions paving way for many unexpected mental traumas due to the panic caused by overthinking. All the social media platforms are jammed with back to back broadcasting of the prevailing pandemic situation. This constant cannonade may have high consequence in intensified anxiety with instant effects on cognitive health [8]. The constant threat feel may result in psychological disorders owing to some extremely developed responses to sickness, fright and the threat of disease can also make us become more sceptical to strangers. Psychological effects of quarantine may differ from instantaneous effects for instance fear of infecting other family members, rejection and avoidance, hopelessness and socio-economic suffering, marking people with stigmata and defaming [9].

\section{Consequences Faced by Assorted People in the Society}

\section{In Kids, Toddlers and Youngsters}

A very critical but seemingly unnoticed matter of concern is the psychological force of COVID-19 occurrence on kids and youngsters. For cerebral, emotional and psychosocial

Fig. 1 COVID cases: a survey

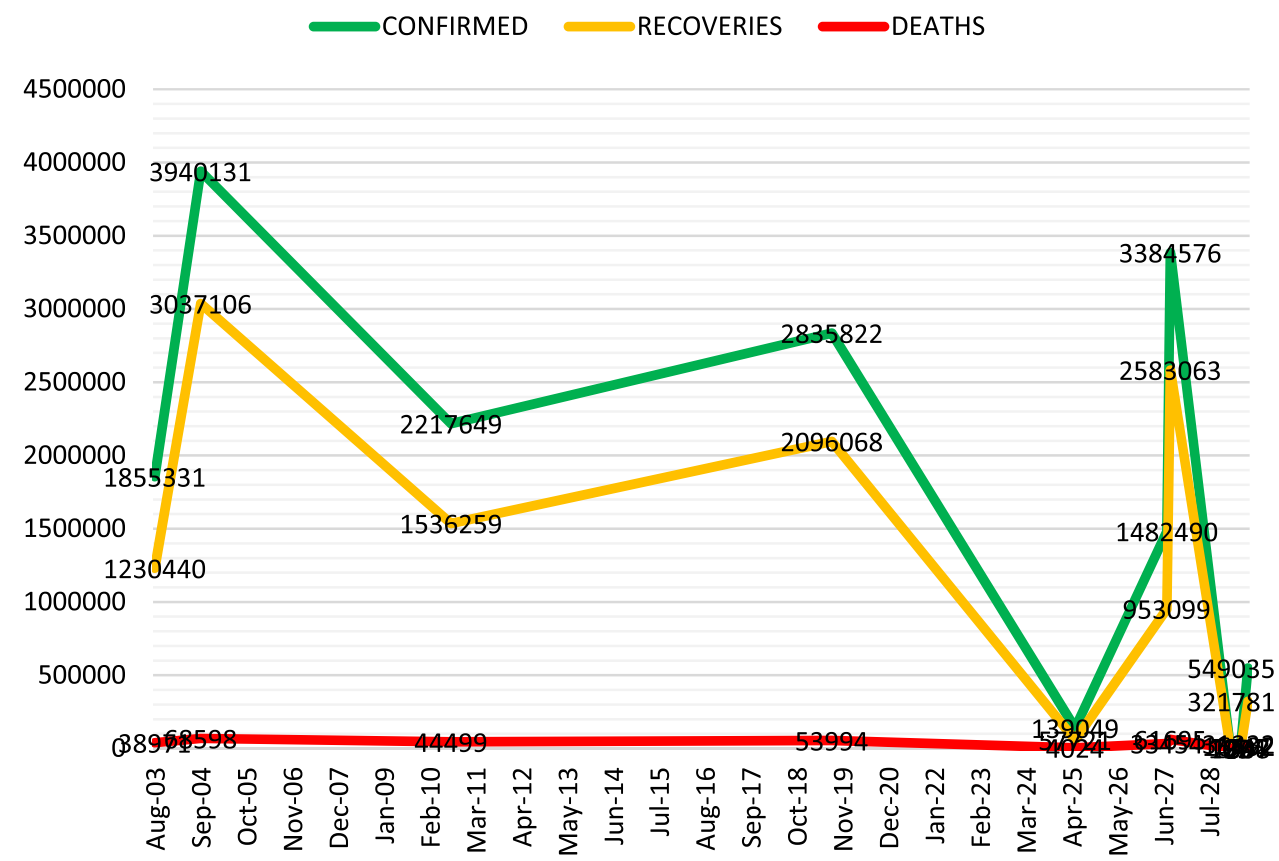


skill development environmental elements in these phases arouse the basic behaviors for lifetime success [10]. During extreme situations like the prevailing pandemic many problems are faced by the young minds like suspending schools, parks and other entertainment zones which disturbs the regular life habits of the children leading to perplexity, intolerance, irritation, frustration, aggression which consecutively causes hostility. These factors automatically provoke mental illness [11]. The parent-child severance may cause uneasiness and worry for themselves and their cherished ones and increase in extended emotional collision.

\section{Elderly Community}

Aged people and people who have severe diseases are particularly susceptible to poorer outcomes from COVID-19 and produce substantial fright among them. Elder adults with cognitive refuse possibly will develop a great deal of unease, trouble, and become generally reserved, therefore their definite wants insist precise notice [12]. Physical workout may be a probable treatment to preserve a healthy bodily wellbeing, and moreover to counter the psychosomatic impact in this tough time [13].

\section{Frontline Workers}

Healthcare workers were expected to experience more psychiatric disorders. The novel coronavirus outbreak threatens to aggravate job-related strain in the midst of some healthcare personnel and to increase their psychological suffering [14]. Extreme fear regarding COVID-19 can have an effect on the capacity and flexibility of folks when they care for moody people at their home as they were performing duties before the occurrence of the epidemic which may inculcate fear, weariness, and abandonment leading to emotional disturbances among them [15].

\section{Disparaged Group of People}

In places with the maximum occurrence of COVID-19 live the huge mass of the world's emigrant and global nomad people. These people are unreasonably exposed to elimination, disgrace and inequity. They contain an elevated occurrence of general psychiatric problems such as sadness, poor standard of living which can be endangered due to quarantine rules forced by the government and loss of job and income during the COVID-19 [5]. It has made the major lockdown occur so as to strictly develop unhappiness in the migrant staff, daily wagers and slum residents globally. Unemployment leads these people incapable to manage life and this unexpected bad luck of poverty, job loss and low income adds to their remorse, annoyance, gloominess and mental agony, eventually leading to functional injury.

\section{Extensive Nervousness in Common People}

An unsure feeling of the future owing to the current lockdown constraint may result in unusually increased unease. Earlier researches have examined the severity and largescale psychosocial complications that these types of contagions can impose on the inhabitants. Owing to the uncertainty and changeable track of the infection, allergy of vagueness, supposed danger of building up the infection can provoke pessimistic emotional feelings together with non-adaptive etiquettes, emotional anguish and isolation amongst people [16]. News about the initial death, expediting in number of latest cases and unrestrained channel awareness can also enhance people's qualms about the situation during disease. This ends up in mislaid health-protection and assisting habits by the common people which may leads to disputes between medical practitioners and patients, which may be destructive to pandemic controlling programmers and hinder steadiness in the society [17].

\section{‘Homeless’ Home-Quarantine}

A large number of people in countries like India face a major problem of homelessness hence the control measures for COVID 19 expected from the public, like isolation, improved sanitation, home-confinement, and stern social distancing are not practically possible among destitute people. Several people experience persistent mental disease, cruelty, difficulties in receiving reasonably priced health care and advanced transience which might become fan the flames in this grave phase of life [5]. The solitary confinement and disease control measures possibly can be confirmed to be unfavorable on the mental illness of homeless public who have unease and scare of compelled hospital stay and thus their rights of this impoverished part of the society remains unconcerned [18].

\section{Pre-existing Psychiatric Infirmity and COVID-19}

People with mental illness are significantly more expected to be tainted by the illness and are in higher danger encountering more physical and psychological pressures during this terminal period. There are more chances for such people to obtain infection due to lack of knowledge, abridged concern on personal cleanliness [19]. And when psychological anguish accompany it becomes more exigent. Health cautious people misconstrue nontoxic physical symptoms as the indication of the hazardous infection which obviously widens their fear and distress [20]. 


\section{Effect of COVID-19: Risk of Anxiety, Depression and Others}

Massive psychological impacts have been aroused in the alarming pandemic period. Pervasiveness of anxiety and depression happens to be highest at patients who tested positive, frontline workers especially health care providers and also among the general public [21]. Stress is something we face in our daily life. It comes in many forms like occupational stress or monetary stress, to social problems, to new life changes, to inner experiences. Traumatic stress throws back revelation to dreadful events usually apart from regular human experiences which are painful emotionally, extreme and stressful. Inexorable loneliness, annoyance, weariness, hysterical fears are also some of the effects caused [22].

\section{Self-Mutilation and Suicide}

Even though there is an increase in prodrome of fretfulness and surviving amidst the constant worry and stress, there is a major threat of self-harm and suicide predominantly among insecure folks [23]. Moreover, with the forced or compelled isolation to reduce the infection the closely connected people of the affected person is unable to identify and impede the suicidal attempt. There is also increase in self-harm due to the vagueness brought about by the pandemic. People's top concerns were the fear of job loss and financial insecurity. Continuous exposure to news of death and destruction can due to Corona virus may boost up the fear and also reinforce suicide risk [24].

\section{Gender Disparities}

Though it is men who often step out of the house and are considered as the bread winners of the family and are likely suspected to bring the infection into their homes. Womenthe caregivers-are the mostly affected persons psychologically when children and aged are at peril. Working women face even more difficulties enduring workplace responsibilities too. At the outset all these factors add stress to women's lives.

\section{Counselling Using AI}

The coronavirus disease and its instantaneous consequences present a severe vulnerability to the mental health of the people resulting in anxiety, depression, stress, and suicidal behaviours. People also face these mental illnesses due to the isolation which threatens them the most. This being the case these quarantined patients cannot avail treatment by visiting a doctor and medical practitioner or a counsellor to let out what's running in their mind. One of the supreme methods is technological or virtual psychiatry treatment applying artificial intelligence [6]. AI has the capability to examine data, and identify patterns to solve or render proper therapy to the particular mental disorder. Chatbots are more human like. It is being used productively as a way of providing useful information exclusively in mental health during this pandemic period which restricts one on one counselling. An interactive chat bot can be created [25].

The main aim of counselling is to bring down negative thoughts and instill comfort, happiness and welfare among people who are really in need of a person whom they can totally rely on but unfortunately friends or family cannot render their shoulders to the affected person due to the prevailing pandemic and more over there will always be a restraint when sharing personal or embarrassing things. Thus a virtual counsellor can be replaced to share things easier and articulate freely by avoiding the feel of being judged.

Using artificial intelligence, it is also possible to spot even facial expressions or nonverbal body language which can draw together all the exteroceptive behaviours and evaluate the mental problem and can deliver a proper counselling to recover from their mental illness.

\section{Starring Role of Al in Counselling}

In building up a system with artificial intelligence, the deep learning approaches are mainly used to muddle through the human intelligence and specifically verbal/nonverbal language is one of the perilous modes through which the patient with mental illness can be tracked even with elusive changes. The virtual counsellor should be designed with intelligence so that it can track it and respond intelligently to the extent where even the Medical advisor is not necessary all the times and our virtual counsellor could be a prime option. Moreover, the diagnostic tool [26-29] in the intrusion of artificial intelligence through healthcare analysis is a great fortune for doctors in many ways also. The data must be preserved with great confidentiality [30] being the right of a human, through various recent techniques.

The system is trained and tested to respond intelligently to shambolic speeches/texts for which Tokenization is used effectively where the alerted data are figured out efficiently. This process is carried out by taking the sentence first through Sentence Tokenize and then words through Word Tokenize, further through Regular expression excruciate the strings into substrings using Regexp Tokenizer and finally even the empty lines are preserved using Blank line Tokenizer. 
Fig. 2 Proposed methodology

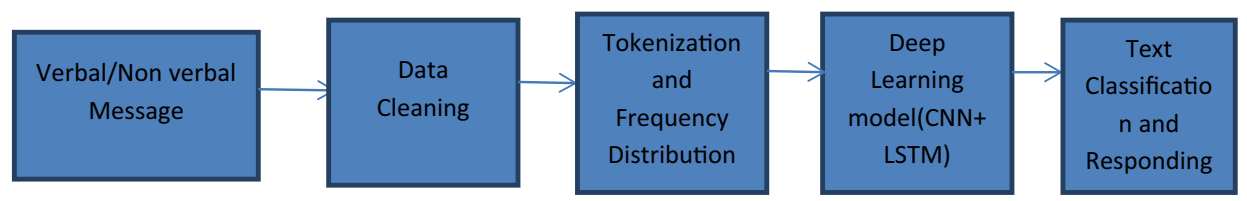

The frequency distribution is elucidated by counting the occurrence of words through which auto encoding is done where the deep learning techniques are extensively used and eventually, there are no possibilities that the response from the Counsellor will never go wrong and the prediction rate would be greatly appreciated. Any model can fit the data but the suggested hybrid model is the combination of LSTM (Long Short term Memory network)and CNN (convolutional neural network) which can seize the local and global features of words and sentences in the text through natural language processing (NLP) for text classification.

The input message from the mentally ill is fed to the system for NLP (Natural Language processing) for data cleaning and the distribution of the frequency of words are categorised and further fed to the deep learning hybrid model for classifying the text and question-aware passage representation and response as shown in Fig. 2. In this multilayer network, information flows layer by layer and thus the auto encoding predicts the words with high accuracy and the performance evaluation is done through the following dominant parameters:

(i) Recognition rate $=$ True positive + true negative/positive + negative

(ii) Misclassification rate $=$ False positive + false negative/ positive + negative

(iii) Sensitivity $=$ True positive/positive

(iv) Specificity $=$ True negative/negative

(v) Precision $=$ True positive/true positive + false positive

\section{Conclusion}

These adverse days may have a deadly effect on the emotional fitness and physical well-being of patients. The best way ahead to support the affected patients is by providing psychological and personal support, relaxation therapy, and psychotherapy services tenuously which aids in reducing anxiety and depression among the affected people. It is also equally sensible to offer community assistance for those living solitary and encourage families and friends to constantly speak to them over phone in-order to reduce emotional isolation and aids in lessening some stress. The psychological impact of fear and anxiety induced by the quick spread of pandemic has to be obviously acknowledged as health precedence for both authorities and strategy makers who should quickly espouse very clear behavioural strategies to reduce the pandemic and the mental depression and other traumas caused. Taking care of such people by friends and family through phone call and video calls makes the society strong mentally. Appropriate mental health care needs have to be developed urgently both in urban and residential areas. Artificial intelligence can also be incorporated along with the clinicians and can provide online counselling with all the reports generated by the virtual counsellor. With the help of artificial intelligence in the field of mental and behavioural health it becomes more opportune and easily approachable.

\section{Declarations}

Conflict of interest The authors declare that they have no competing interests.

\section{References}

1. Wang C, Pan R, Wan X, Tan Y, Xu L, McIntyre RS, Choo FN, Tran B, Ho R, Sharma VK, Ho C. A longitudinal study on the mental health of general population during the COVID-19 epidemic in China. Brain Behav Immun. 2020;87:40-8.

2. Hossain MM, Sultana A. NPurohit. Epidemiol Health. 2020;42:1-11.

3. Harper CA, Satchell LP, Fido D, Latzman RD. Functional fear predicts public health compliance in the COVID-19 pandemic. Int J Ment Health Addict. 2021;19(5):1875-88.

4. Chatterjee K, Chauhan VS. Epidemics, quarantine and mental health. Med J Armed Forces India. 2020;76(2):125-7.

5. Dubey S, Biswas P, Ghosh R, Chatterjee S, Dubey MJ, Chatterjee S, Lahiri D, Lavie CJ. Psychosocial impact of COVID-19. Diabetes \& Metabolic Syndrome: Clinical Research \& Reviews. 2020:14(5);779-88.

6. Ćosić K, Popović S, Šarlija M, Kesedžić I, Jovanovic T. Artificial intelligence in prediction of mental health disorders induced by the COVID-19 pandemic among health care workers. Croat Med J. 2020;61(3):279-88.

7. Chatterjee P, Nagi N, Agarwal A, Das B, Banerjee S, Sarkar S, Gupta N, Gangakhedkar RR. The 2019 novel coronavirus disease (COVID-19) pandemic: a review of the current evidence. Indian J Med Res. 2020;151(2):147-59.

8. Mertens G, Gerritsen L, Duijndam S, Salemink E, Engelhard IM. Fear of the coronavirus (COVID-19): Predictors in an online study conducted in March 2020. J Anxiety Disord. 2020:74;102258.

9. Pérez-Fuentes MDC, Molero Jurado MDM, Martos Martínez Á, Gázquez Linares JJ. Threat of COVID-19 and emotional state during quarantine: Positive and negative affect as mediators in a cross-sectional study of the Spanish population. PLOS ONE. 2020:15(6); 0235305 . 
10. Jiao WY, Wang LN, Liu J, Fang SF, Jiao FY, Pettoello-Mantovani M, Somekh E. Behavioral and emotional disorders in children during the COVID-19 epidemic. J Pediatr. 2020:221;264-6.

11. Jefsen $\mathrm{OH}$, Rohde $\mathrm{C}, \mathrm{N} \emptyset$ rremark B, Østergaard SD. Editorial Perspective: COVID-19 pandemic-related psychopathology in children and adolescents with mental illness. J Child Psychol Psychiatry. 2021:62(6);798-800.

12. Vahia IV, Blazer DG, Smith GS, Karp JF, Steffens DC, Forester BP, Tampi R, Agronin M, Jeste DV, Reynolds CF III. COVID-19. Pmc J. 2020;28(7):695-7.

13. Jiménez-Pavón D, Carbonell-Baeza A, Lavie CJ. Physical exercise as therapy to fight against the mental and physical consequences of COVID-19 quarantine: Special focus in older people. Prog Cardiovasc Dis. 2020:63(3):386.

14. Spoorthy MS, Pratapa SK, Mahant S. Mental health problems faced by healthcare workers due to the COVID-19 pandemic-a review. Asian J Psychiatry. 2020;51:102119.

15. Williamson V, Murphy D, Greenberg N. COVID-19 and experiences of moral injury in front-line key workers. Occup Med. 2020;70(5):317-9.

16. Ornell F, Schuch JB, Sordi AO, Kessler FH. "Pandemic fear" and COVID-19: mental health burden and strategies. Braz J Psychiatry. 2020;42:232-5.

17. Bashir MF, Shahzad K, Komal B, Bashir MA, Bashir M, Tan D, Fatima T, Numan U. Environmental quality, climate indicators, and COVID-19 pandemic: insights from top 10 most affected states of the USA. Environ Sci Pollut Res Int. 2021;28(25):32856-65.

18. Tsai J, Wilson M. COVID. Lancet J. 2020;5(4):186-7.

19. Asmundson GJ, Paluszek MM, Landry CA, Rachor GS, McKay D, Taylor S. Do pre-existing anxiety-related and mood disorders differentially impact COVID-19 stress responses and coping? J Anxiety Disord. 2020:74;102271.

20. Chatterjee SS, Vora M, Malathesh BC, Bhattacharyya R. Worried well and Covid-19: Re-emergence of an old quandary. Asian J psychiatry. 2020:54;102247.

21. Huang Y, Zhao N. Corrigendum to Generalized anxiety disorder, depressive symptoms and sleep quality during COVID-19 outbreak in China: a web-based cross-sectional survey. Psychiatry Res. 2021:288(2020);112954.
22. Wang C, Pan R, Wan X, Tan Y, Xu L, Ho CS, Ho RC. Immediate psychological responses and associated factors during the initial stage of the 2019 coronavirus disease (COVID-19) epidemic among the general population in China. Int J Environ Res Public Health. 2020:17(5);1729.

23. Montemurro N. Intracranial hemorrhage and COVID-19, but please do not forget "old diseases" and elective surgery. Brain Behav Immun. 2021;97:207.

24. Courtet P, Olié E, Debien C, Vaiva G. Keep socially (but not physically) connected and carry on: preventing suicide in the age of COVID-19. J clin Psychiatry. 2020;81(3):15527.

25. Cameron G, Cameron D, Megaw G, Bond R, Mulvenna M, O’Neill S, Armour C, McTear M. Towards a chatbot for digital counselling, Proceedings of the 31st International BCS Human Computer Interaction Conference; 2017.

26. IgnishaRajathi G, WiselinJiji G. A novel automatic liver segmentation by level set method over real-time sensory computed tomography. Wirel Pers Commun. 2019;109:1987-2010. https:// doi.org/10.1007/s11277-019-06664-9.

27. IgnishaRajathi G, WiselinJiji G. Chronic liver disease classification using hybrid whale optimization with simulated annealing and ensemble classifier. Symmetry. 2019;11:33. https://doi.org/ 10.3390/sym 11010033 .

28. Vedhapriyavadhana R, Sneha C, Shalini A, Subashinikrishna S. Myocardial analysis using deep learning neural network. J Interdiscip Cycle Res. 2020;12(3):594-603.

29. Niranjana R, Ravi A, Vedhapriyavadhana R, Francy I, Rani E, Prasanth NN. Breast cancer detection using deep learning neural network with image processing techniques. Solid State Technol. 2020;63(5):4947-55.

30. Nagajothi S, Ignisha Rajathi G, Manikandan M, Boopala J. Data privacy preservation in cloud using Mapreduce. Int J Sci Technol Res ISSN No. 2277-8616, February 2020, Volume 9, Issue 2.

Publisher's Note Springer Nature remains neutral with regard to jurisdictional claims in published maps and institutional affiliations. 